\title{
Control of MHD Instabilities by ECCD: ASDEX Upgrade Results and Implications for ITER
}

\author{
H. Zohm 1), G. Gantenbein 2), F. Leuterer 1), A. Manini 1), M. Maraschek 1), Q. Yu 1) \\ and the ASDEX Upgrade Team
}

1) MPI für Plasmaphysik, D-85748 Garching, Germany, EURATOM Association

2) Forschungszentrum Karlsruhe, IHM, D-76021 Karlsruhe, Germany, EURATOM Association

e-mail contact of main author: zohm@ipp.mpg.de

\begin{abstract}
The requirements for control of MHD instabilities by ECCD are reviewed. It is shown that localised current drive is needed for control of both sawteeth and Neoclassical Tearing Modes (NTMs). In the case of NTMs, the deposition width should be smaller than the island width for efficient control. At island widths smaller than the deposition width, as is predicted to occur in ITER, theory suggests that efficient control is possible only by modulating the ECCD power in phase with the island. These predictions are experimentally confirmed in ASDEX Upgrade for NTM control. Narrow deposition has also been used to extend the operational range of NTM stabilisation in ASDEX Upgrade to lower $q_{95}$ and in the improved H-mode scenario. Our results suggest that for the ITER ECCD system, good localisation of the driven current profile as well as the capability to modulate the ECCD in phase with rotating modes will be needed for efficient MHD control by ECCD.
\end{abstract}

\section{Introduction}

MHD instabilities limit the operational space of tokamaks in several ways [1]. Their control is therefore of great interest to expand operational space and hence the performance of present day and future tokamaks, such as ITER. Electron Cyclotron Current Drive (ECCD) with its highly flexible, localised deposition is an ideal tool for this purpose. Based on recent success in the area of control of sawteeth [2], [3] and Neoclassical Tearing Modes (NTMs) [4], [5], [6], ECCD is also foreseen as an MHD control tool in ITER. Hence, it is important to expand our physics base of MHD control by ECCD and to verify the control strategies proposed for ITER in present day experiments.

ASDEX Upgrade, a medium size divertor tokamak $\left(R_{0}=1.65 \mathrm{~m}, a=0.5 \mathrm{~m}\right)$ with an ITERlike cross-section (single-null divertor, elongation up to 1.8 , triangularity up to 0.5 ) and a versatile heating system, including a $140 \mathrm{GHz}$ ECRH system with at present more than $2 \mathrm{MW}$ of installed power, has a strong programme in this area [7], focussed directly on ITER needs. In this paper, we point out the requirements arising from the present theoretical understanding of the control of sawteeth and NTMs by ECCD and then present recent ASDEX Upgrade results that verify the basic predictions from theory. Finally we will also point out the implications of these findings for ITER.

\section{Requirements for MHD mode control by ECCD}

Control of MHD instabilities by ECCD is based on the generation of a localised current at the mode rational surface of interest. Stability can be altered due to the change of equilibrium current profile or due to the generation of a helical current within the island associated with a 
resistive MHD mode. For the sawtooth instability, the criterion to influence stability by changing the equilibrium current profile, and thus altering the shear at the $q=1$ surface, through an ECCD driven current $I_{E C C D}$ has been estimated to be [8]

$$
I_{E C C D}>2\left(d / r_{q=1}\right)^{2} I_{q=1}
$$

where $d$ is the 1/e radial half width of the current distribution added by ECCD and $I_{q=1}$ is the total plasma current inside the $q=1$ surface. We note that this criterion does not take into account the effects of fast particles on sawtooth stability, so that it will not apply quantitatively to ITER, but we expect it to be qualitatively describing the situation there as well. It can be seen that this criterion emphasizes the need to localize the ECCD current as the figure of merit for given equilibrium is $I_{E C C D} / d^{2}$. The same is true for stabilising classical or neoclassical tearing modes by altering the equilibrium current profile ( $\Delta^{\prime}$ effect) as can be seen from the Rutherford equation for the island width $W$

$$
\frac{\tau_{r e s}}{r_{s}} \frac{d W}{d t}=a_{b s} r_{s} \beta_{p} \frac{1}{W}-r_{s} \Delta_{s t a b}^{\prime}-\frac{L_{q} r_{s}}{d^{2}} \frac{I_{E C C D}}{I_{p}\left(r_{s}\right)}\left(a_{m n} \eta_{m n}(W / d) \frac{d^{2}}{W^{2}}+a_{00}\right)
$$

where the first term on the right hand side is the destabilizing bootstrap current contribution, the second term contains all intrinsic stabilizing terms such as e.g. the effect of the equilibrium current profile $\left(\Delta^{\prime}\right)$, the finite parallel heat conductivity or the polarization current and the last term is the ECCD contribution (for a more complete discussion, see e.g. [9]). In this last term, the second term in the bracket is the contribution to the equilibrium current profile, again proportional to $I_{E C C D} / d^{2}$.

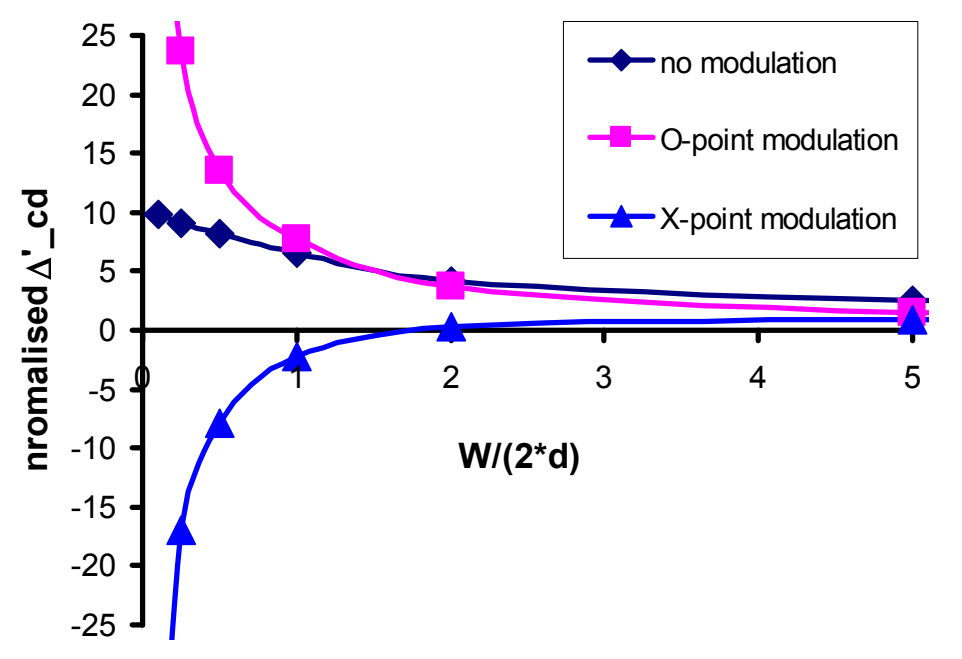

FIG 1. The function $I_{E C C D}\left(a_{m n} \eta_{m n}(d / W)^{2}+a_{00}\right)$, relating to the stabilizing term in Eqn. (2) plotted for unmodulated $\left(I_{E C C D}=1\right)$ current drive as well as modulation $\left(I_{E C C D}=0.5\right)$ around the O-point and the Xpoint of the island (50\% duty cycle, $100 \%$ modulation depth).

In Eqn. (2), we distinguish between the equilibrium $(0,0)$ component of the ECCD driven current and the helical $(m, n)$ component. The efficiency of generating such a helical current component has been taken into account in the function $\eta_{m n}$, which must be calculated by averaging the RF source profile $j(r, \zeta)=j_{E C C D} \exp \left(-(r / d)^{2}\right) \Theta(\zeta)$, where $\Theta(\zeta)$ characterizes the modulation in helical angle, over the island flux surfaces as in [10]. As has been pointed out 
e.g. in [10], $\eta_{m n}$ decreases when $d$ becomes bigger than $W$. For DC current injection, we then have $\eta_{m n} \sim(W / d)^{2}$ and the stabilisation term becomes constant whereas the bootstrap drive still goes like $1 / W$. However, it is predicted that this can be partly recovered by modulating the ECCD source in phase with the O-point of the island, because then, $\eta_{m n} \sim(W / d)$.

To illustrate this, we have plotted in Fig. 1 the function $\Delta_{C D}^{\prime}=I_{E C C D}\left(a_{m n} \eta_{m n}(d / W)^{2}+a_{00}\right)$ as function of $W /(2 d)$, i.e. full island width normalized to the full $1 / \mathrm{e}$ width, for the modulated (50\% duty cycle, $100 \%$ modulation depth, O-point and X-point modulation) and the unmodulated case using the literature values $a_{m n}=32$ [10] and $a_{00}=2$ [11]. Due to the assumption that the $(0,0)$ term is independent of $W$, it dominates for large $W$ and makes the unmodulated case exceed the modulated case. For small $W /(2 d)$, the $(m, n)$ component dominates and in this regime, O-point modulation exceeds the unmodulated case. For X-point modulation, the helical current is destabilizing, whereas the $(0,0)$ component is stabilizing. Thus, for small $W /(2 d), \Delta_{C D}^{\prime}$ becomes negative whereas for large $W /(2 d)$ it is positive. We note however, that the analytical form of the stabilising effect of the $(0,0)$ component in Eqn. (2) is derived under the assumption that $W<2 d$, so that a more careful analysis will have to be done in future work. In the next sections we will discuss ASDEX Upgrade experiments that address both the issue of narrow deposition and modulation.

\section{Sawtooth tailoring}

Previous experiments on sawtooth tailoring of plasmas with dominant NBI heating had been performed with relatively broad ECCD deposition of $d / a=0.05$, where $a$ is the plasma minor radius [3]. In these experiments, a clear stabilisation (i.e. longer sawtooth period $\tau_{S T}$ ) had been found with co-ECCD just outside the $\mathrm{q}=1$ surface and a shorter sawtooth period with coECCD inside $q=1$, both findings consistent with a change in local shear there. However, ctrECCD had shown less pronounced effect, at that time explained by the opposite signs of the contribution from heating with respect to ctr-ECCD.
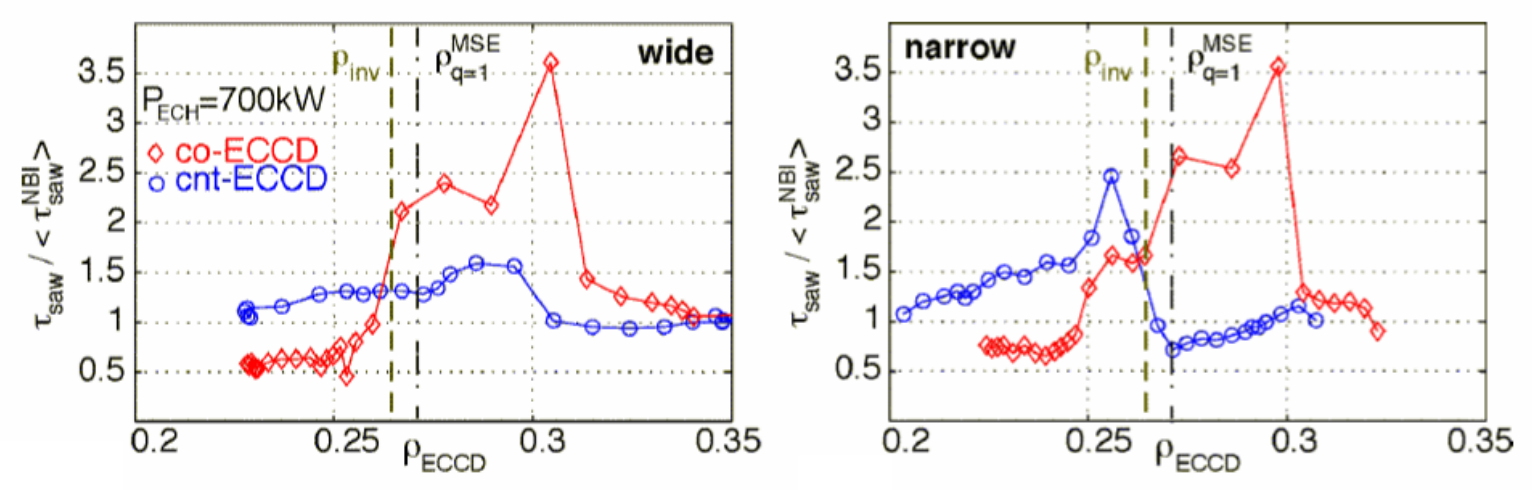

FIG. 2: Dependence of sawtooth period $\tau_{\text {saw }}$, normalised to the sawtooth period with pure NBI heating $\tau_{\text {saw }}{ }^{N B I}$ in an $H$-mode discharge with $q_{95}=4.5$. The variation of the deposition was done by slow $B_{t^{-}}$ ramps. The ECCD power of $0.8 \mathrm{MW}$ was small compared to the auxiliary NBI power of $P_{N B I}=5 \mathrm{MW}$.

A comparison between the previous findings and more recent experiments [12] using narrower deposition $(d / a=0.02)$ is shown in Fig. 2. In these experiments, the current density is roughly doubled with narrow deposition while the heating power is constant. We thus expect the current drive contribution to be dominating over the heating effect. This can in fact be concluded from the right part of Fig. 2 where we now notice a clear stabilising effect with 
ctr-ECCD inside $q=1$ and a less pronounced, but detectable, destabilising effect of ctr-ECCD outside $q=1$. For CD further inside $q=1$, where a shortening of the sawtooth period is observed, the narrow deposition does not seem to have an advantage over the wide deposition, which may be expected when the change of shear is rather global than local (criterion (1) was derived for CD just at the resonant surface, not well inside). We note that, as pointed out in Section 2, in order to quantitatively extrapolate the usefulness of the sawtooth control experiments by ECCD, the effects of fast particles on sawtooth stability, which are expected to be dominant in ITER but not in these ASDEX Upgrade discharges, have to be taken into account.

We also note that the long sawtooth periods obtained in these experiments at least show that we are close to complete stabilisation with these parameters; this is quite consistent with the criterion given in Eqn. (1) for the broad profile, where (1) predicts a requirement of $9 \mathrm{kA}$ and $I_{E C C D}$ from TORBEAM is $10 \mathrm{kA}$, whereas for the narrow deposition, (1) predicts $1.4 \mathrm{kA}$ and $I_{E C C D}=7.5 \mathrm{kA}$. Thus, an increase in stabilisation efficiency as would be predicted from Eqn. (1), where $I_{E C C D} / d^{2}$ goes up by almost a factor of 4, seems not to be not visible. However, one has to keep in mind that the deposition is varied in these experiments by a slow $B_{t}$ ramp which moves the deposition by $3.5 \mathrm{~cm} / \mathrm{s}$, i.e. the maximum moves by one half width in about 300 ms. Hence, at the longest sawtooth periods observed here $(200 \mathrm{~ms})$, we thus do no longer have quasi-static conditions for the corresponding data point. Further experiments with a discharge-to-discharge variation of $B_{t}$ will thus be undertaken to clarify this point.

\section{NTM control experiments with variable deposition width and modulated injection}

The beneficial effect of narrow deposition has been documented for NTMs on ASDEX Upgrade before [7]. Fig. 3 summarises a series of experiments on this subject where by varying the toroidal injection angle, we have varied $I_{E C C D} / d$ in $(3,2) \mathrm{NTM}$ stabilisation experiments at similar plasma parameters $\left(I_{p}=0.8 \mathrm{MA}, B_{t}=2.1 \mathrm{~T}\right)$ [13]. The normalisation accounts for variations in the $\mathrm{CD}$ effciency due to slight variations of local plasma parameters. It is clear that the figure of merit $H_{\text {withECCD }} / H_{w / O E C C D}$, characterising the increase in confinement factor due to reduction or complete stabilisation of the mode increases with better localisation of the ECCD current.

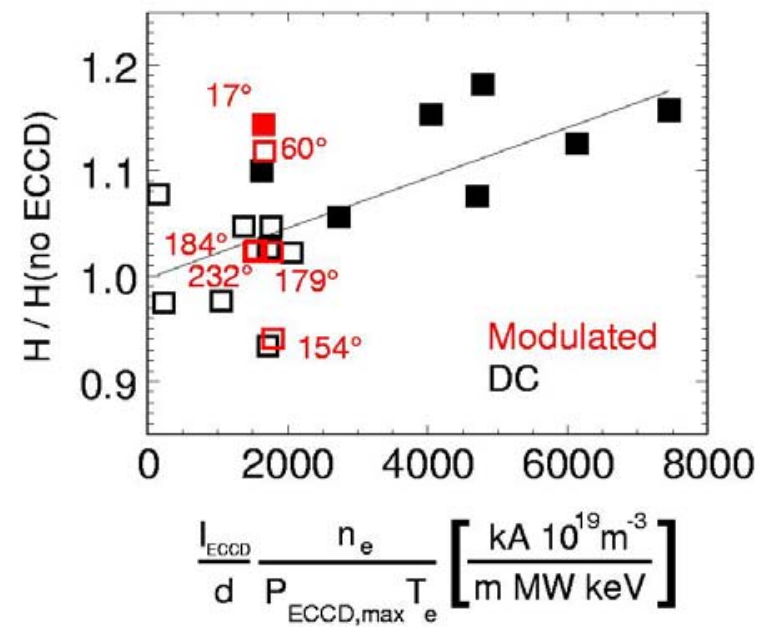

FIG.3: The relative increase in confinement factor $H_{98}$ due to $(3,2)$ NTM stabilisation for a series of otherwise identical discharges under variation of the deposition width, leading to a variation of peak current density driven by ECCD. Black points indicate DC injection, red points are for modulated injection. The red numbers indicate the phase of the modulated ECCD relative to the O-point. Full symbols refer to complete stabilisation; open symbols to partial stabilisation. The straight line is a linear fit to the unmodulated points.

In Fig. 3, we have also inserted the results from experiments where the ECCD power was modulated with $50 \%$ duty cycle and close to $100 \%$ modulation depth. The numbers indicate the phase relative to the O-point of the island. For injection close to the O-point $\left(17^{\circ}\right)$, they reach the highest values of this subset whereas X-point modulation reduces the efficiency 
below that of DC injection. One has to note that with modulation, only half of the ECCD power was injected on average (albeit at same $P_{\max }$ ), proving that with modulation, the power can be used more efficiently (although the installed peak power has to be the same for both schemes). This is consistent with the previous finding at narrow deposition width $W /(2 d) \geq 1$ where no substantial difference between DC and modulation using the same maximum ECCD power (and hence only half the average power in the modulated case) was found [4].

These experiments were done at a toroidal injection angle of $19^{\circ}$, leading to a broad deposition of $W_{\text {marg }} /(2 d)=0.6$ (in the experiment, the lowest value of $W$ at which ECCD power has to be injected is $W_{\text {marg }}$, the so-called marginal island width below which the NTM decays on its own). With that broad deposition, complete stabilisation cannot be achieved any longer using DC injection at the ECCD power used in these experiments (1.1 MW).
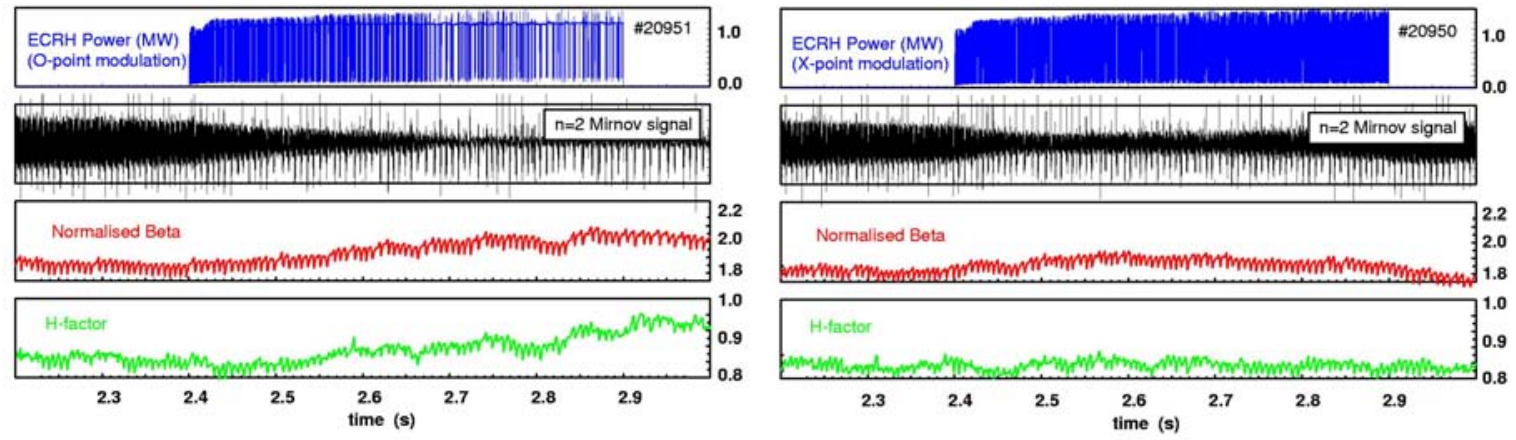

FIG. 4. Stabilisation experiment of a $(3,2)$ NTM by sweeping ECCD over the $q=1.5$ surface using a $B_{t}$ ramp with a broad deposition profile $\left(W_{\text {marg }} /(2 d)=0.6\right)$. Modulated ECCD around the O-point leads to complete stabilization (left) whereas modulated ECCD around the X-point only slightly reduces the mode amplitude (right).

On the other hand, as shown in the left part of Fig. 4, complete stabilisation can be achieved with modulated injection around the O-point of the island under otherwise identical conditions. After stabilisation, the ECCD power switches to DC, with interruptions due to ELMs producing trigger signals. Even after turning off the ECCD, the mode does not come back, because there is no large sawtooth to trigger it, which would be required at the reltively modest value of $\beta$. In the right part of Fig. 4, we also show an experiment using modulated injection around the X-point. This shows a small stabilising effect, probably because the $\Delta^{\prime}$ effect dominates over the negative helical current that is created by the modulated injection around the X-point. This is also consistent with the positive values of the efficiency function for X-point modulation in Fig. 1 at large values of $W /(2 d)$ (for the saturated island in Fig. 4, we estimate $\left.W_{\text {sat }} /(2 d) \approx 2.5\right)$.

\section{Extension of parameter space for NTM stabilisation}

The experiments shown above have, as almost all previous NTM stabilisation experiments in ASDEX Upgrade for both $(3,2)$ and $(2,1)$ NTMs, been carried out in a standard H-mode scenario with relatively high $q_{95}\left(I_{p}=0.8 \mathrm{MA}, B_{t}=2.1\right.$, resulting in $q_{95}$ around 4.5$)$ and at $\beta_{N}$ in the range 1.8-2.3. In this section, we report on new experiments at low $q_{95}$ and in the improved H-mode regime.

Experiments towards stabilisation at lower $q_{95}$ were carried out by increasing the plasma current. In addition, the toroidal field had to be slightly lowered because the resonant surface 
shifts further outside in such a plasma (i.e. towards the centre post on the HFS, where the ECCD deposition is located in these experiments). Fig. 5 shows time traces from a lower $q_{95}$ $\left(I_{p}=1.0 \mathrm{MA}\right.$, resulting in $\left.q_{95}=3.8\right)$ discharge with successful stabilisation using DC injection and optimised narrow deposition. A characteristic feature of the $(3,2)$ mode in lower $q_{95}$ scenarii is that the impact on confinement is generally more pronounced. Consequently, also the rise in $\beta_{N}$ and $H_{98}$ after full stabilisation is stronger than at higher $q_{95}$. We attribute that to the fact that at lower $q_{95}$, the $(3,2)$ rational surface is located further outward in a zone of steeper gradients, thus leading to a stronger impact on the confinement. This is consistent with experimental results from $(2,1)$ NTM stabilisation in ASDEX Upgrade reported previously [2], where it was also found that the increase in $\beta_{N}$ and $H$ was more substantial than for the $(3,2)$ mode (the $(2,1)$ rational surface is located even further outside).

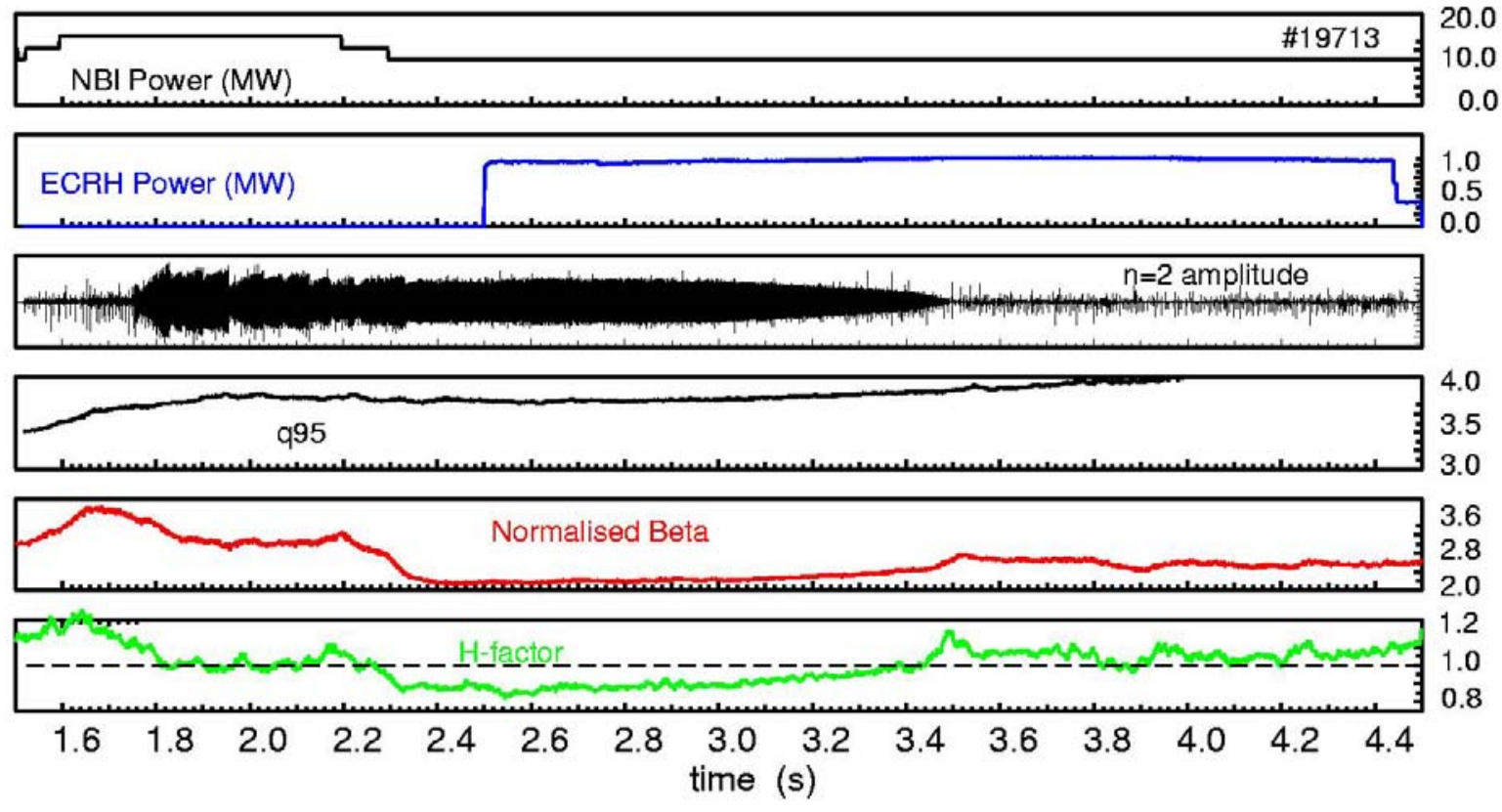

FIG. 5:Full stabilisation of a $(3,2)$ NTM at $q_{95}=3.8$ using narrow deposition $\left(W_{\text {marg }} /(2 d)=\right.$ 1.6). Note the pronounced gain in $\beta_{N}$ and $H_{98}$ after complete stabilisation.

Fig. 6 shows an example of complete stabilisation of a $(3,2)$ NTM at even lower $q_{95}=2.9$ and at $\beta_{N}=2$, i.e. at values very close to the ITER standard operating point which has $q_{95}=3$ and $\beta_{N}=1.8$. 

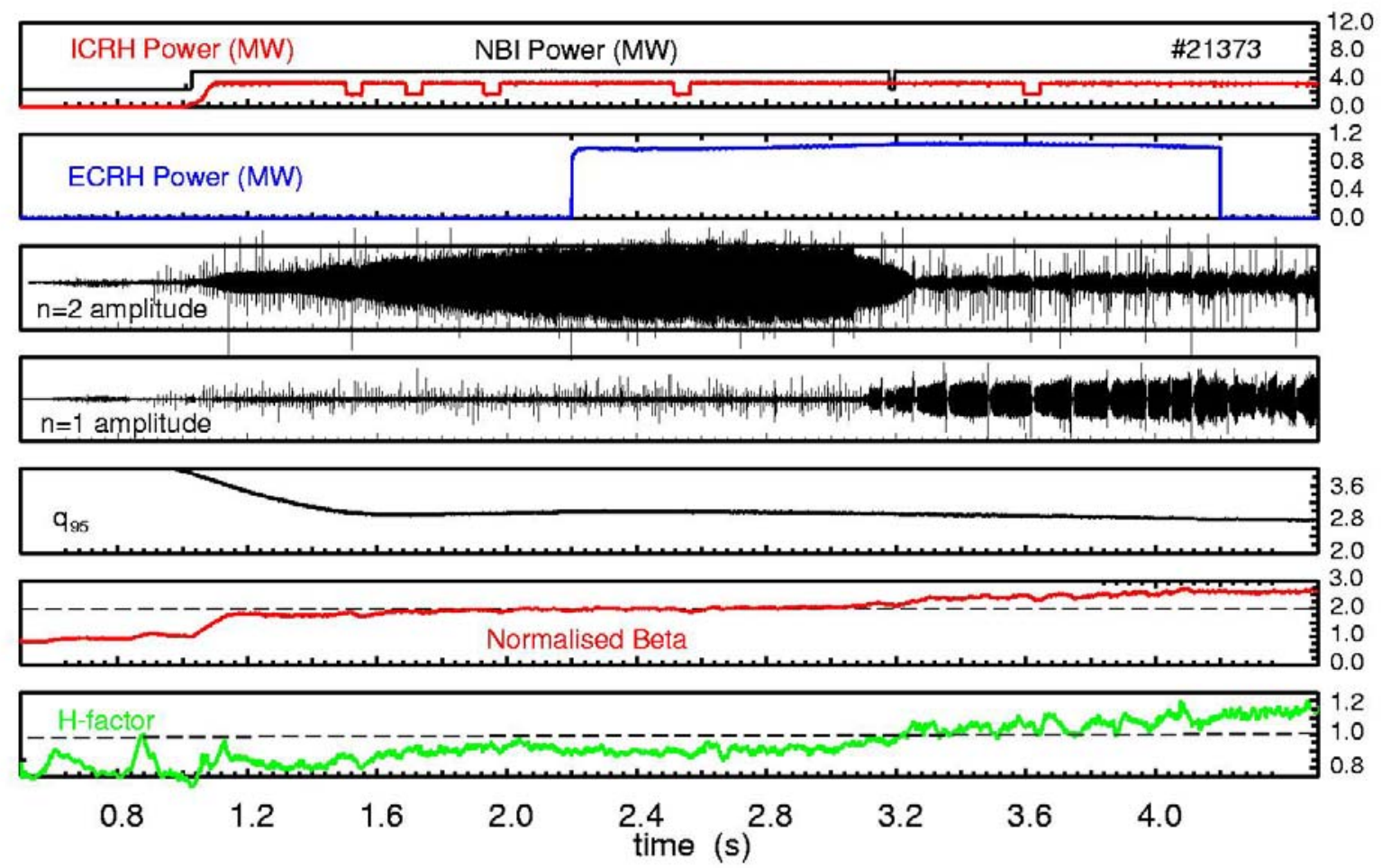

FIG. 6. Stabilisation experiment of a $(3,2)$ NTM by sweeping ECCD over the $q=1.5$ surface using a $B_{t}$ ramp with a narrow deposition profile $\left(W_{\text {marg }} /(2 d)=1.6\right)$ in an improved $H$-mode at $q_{95}=2.9$.

This discharge is performed under conditions for the improved H-mode regime [14], i.e. with early NBI heating during the current ramp to establish a flat, elevated central q-profile avoiding the occurrence of sawteeth. While at higher $q_{95}$, the $(3,2)$ NTM may be beneficial in this regime in that it clamps the q-profile to values not permitting sawteeth at relatively low penalty in confinement, it is clear from Fig. 6 that at that low $q_{95}$, the loss of confinement due to the $(3,2)$ is not acceptable $(H$-factor below 1$)$. However, ECCD stabilisation of the $(3,2)$ mode allows the discharge to enter into good improved H-mode conditions with $H_{89}=1.15$ and $\beta_{N}$ rising from 2 during the $(3,2)$ mode up to 2.6 in the following improved H-mode phase. We note that the self-organisation of the q-profile in this discharge is, as often in ASDEX Upgrade, accompanied by the onset of fishbone activity, as indicated by the trace ' $n=1$ amplitude' in Fig. 6. Stabilisation of the $(3,2)$ seems to be a pre-requisite for this, probably because the presence of the mode redistributes the fast particles such that the drive for fishbones is not sufficient while the $(3,2)$ mode is there.

\section{Summary and Conclusions for ITER}

The efficiency of MHD control by ECCD, which is predicted to increase with more localized ECCD deposition is found to follow the theoretical predictions for NTM stabilisation experiments in ASDEX Upgrade. In particular, the predicted loss of efficiency for cases where the full ECCD deposition width exceeds the island size is found in the experiments. We have also proven experimentally that this loss of efficiency can to some extent be recovered by modulating the ECCD injection around the O-point of the mode. For sawteeth, the effect on the efficiency is less clear experimentally and the subject needs further experimental investigation. 
For the ITER ECCD system, this means that the present optimisation strategy, which uses as a figure of merit the ECCD driven current density, based on theoretical predictions, has now been justified experimentally. The ITER ECCD system, especially the upper launcher, which is aimed at NTM control, should thus be optimised for narrow deposition. In addition, modulation capability of the gyrotrons should be foreseen to enable phased injection into a rotating NTM island at small island width, which is predicted to be the case in ITER because $W_{\text {marg }}$ scales like the normalised poloidal ion gyroradius $\rho_{p i}$ and not with machine size. Concerning the extrapolation of the required power to ITER, we note that the ASDEX Upgrade data have entered into a multi-machine database used to extrapolate the requirements [15]. At present, the ITER system looks adequate for NTM stabilisation if modulation is applied, but further work on the multi-machine database will be needed to confirm this finding.

Finally, we also mention here the next steps undertaken at present on ASDEX Upgrade to verify feedback controlled MHD mode stabilisation. A central part of this is the on-line determination of both MHD mode position as well as ECCD deposition and its use to poloidally steer the ECCD antenna mirrors for deposition control. Such a system is presently being installed in ASDEX Upgrade in the framework of the extension of the ECCD system to $4 \mathrm{MW}, 10 \mathrm{~s}$, with variable frequency [16]. It will permit to test several options of sensors (such as ECE diagnostics, ECE sightline along the ECRH launcher or on-line current profile reconstruction) and actuators (poloidal mirror steering, shift of the radial plasma position) that have been proposed to approach this problem for ITER.

\section{References}

[1] ZOHM, H. et al., "MHD limits to tokamak operation and their control", Plasma Phys. Control. Fusion 45 (2003) A163.

[2] ANGIONI, C. et al., "Effects of localized electron heating and current drive on the sawtooth period", Nucl. Fusion 43 (2003) 455-468.

[3] MÜCK, A. et al., "Sawtooth Control Experiments in ASDEX Upgrade", Plasma Phys. Control. Fusion 47 (2005) 1633.

[4] ZOHM, H. et al., "Experiments on Neoclassical Tearing Mode Stabilisation by ECCD in ASDEX Upgrade", Nucl. Fusion 39 (1999) 577.

[5] LA HAYE, R.J., "Control of Neoclassical Tearing Modes in DIII-D", Phys. Plasmas 9 (2002) 2051.

[6] ISAYAMA, A., "Complete stabilisation of a neoclassical tearing mode in steady state high $\beta_{\mathrm{p}} \mathrm{H}$-mode discharges by the first harmonic electron cyclotron heating/current drive in JT-60U", Plasma Phys. Control. Fusion 42 (2000) L37.

[7] MARASCHEK, M. et al., "Active control of MHD instabilities by ECCD in ASDEX Upgrade", Nucl. Fusion 45 (2005) 1369.

[8] MERKULOV, A. et al., "Sawtooth period control by localized non-inductive current drive", Proceedings of Joint Varenna-Lausanne Int. Workshop on Theory of Fusion Plasmas, Varenna, Italy, (2004) 279. 
[9] ZOHM, H. et al., "Neoclassical Tearing Modes and Their Stabilisation by ECCD in ASDEX Upgrade", Phys. Plasmas 8 (2001) 2009.

[10] HEGNA, CC. and CALLEN, J.D., "On the stabilisation of neoclassical magnetohydrodynamic tearing modes using localised current drive or heating", Phys. Plasmas 4 (1997) 2940.

[11] PLETZER, A. and PERKINS, F.W., "Stabilisation of neoclassical tearing modes using a continuous localised current drive", Phys. Plasmas 6 (1999) 1589.

[12] MANINI, A. et al., "Optimisation of MHD Stability Using ECCD in ASDEX Upgrade", Proc. of the $14^{\text {th }}$ workshop on ECE and ECRH (EC-14), Santorin, Greece (2006).

[13] MARASCHEK, M. et al., "Enhancement of the Stabilisation Efficiency of a Neoclassical Magnetic Island by Modulated Electron Cyclotron Current Drive in the ASDEX Upgrade Tokamak", Phys. Rev. Lett. 98 (2007) 025005.

[14] GRUBER, O., et al., "Stationary H-mode discharges in ASDEX Upgrade with internal transport barrier", Phys. Rev. Lett. 83 (1999) 1787.

[15] LA HAYE, R.J. et al., "Cross-machine benchmarking for ITER of neoclassical tearing mode stabilization by electron cyclotron current drive", Nucl. Fusion 46 (2006) 451.

[16] MANINI, A. et al., "Development of a feedback system to control MHD instabilities in ASDEX Upgrade ", to appear in Fusion Engineering and Design (2006). 